\title{
Sparse Matrix Decomposition with Optimal Load Balancing*
}

\author{
Ali Pinar and Cevdet Aykanat \\ Computer Engineering Department, Bilkent University \\ TR-06533 Bilkent, Ankara, Turkey \\ \{apinar/aykanat\}@cs.bilkent.edu.tr
}

\begin{abstract}
Optimal load balancing in sparse matrix decomposition without disturbing the row/column ordering is investigated. Both asymptotically and run-time efficient exact algorithms are proposed and implemented for one-dimensional (ID) striping and two-dimensional (2D) jagged partitioning. Binary search method is successfully adopted to $1 D$ striped decomposition by deriving and exploiting a good upper bound on the value of an optimal solution. A binary search algorithm is proposed for $2 D$ jagged partitioning by introducing a new $2 D$ probing scheme. A new iterative-refinement scheme is proposed for both $1 D$ and $2 D$ partitioning. Proposed algorithms are also space efficient since they only need the conventional compressed storage scheme for the given matrix, avoiding the need for a dense workload matrix in $2 D$ decomposition. Experimental results on a wide set of test matrices show that considerably better decompositions can be obtained by using optimal load balancing algorithms instead of heuristics. Proposed algorithms are 100 times faster than a single sparse-matrix vector multiplication (SpMXV), in the 64-way $1 D$ decompositions, on the overall average. Our jagged partitioning algorithms are only $60 \%$ slower than a single SpMxV computation in the $8 \times 8-w a y 2 D$ decompositions, on the overall average.
\end{abstract}

\section{Introduction}

Sparse-matrix vector multiplication ( $\mathrm{SpMxV}$ ) constitutes the most time consuming operation in iterative solvers. Parallelization of $\mathrm{SpMxV}$ operation requires the decomposition and distribution of the coefficient matrix. Two objectives in the decomposition are the minimization of the communication requirement and the load imbalance. Graph theoretical approach is the most commonly used decomposition technique in the literature. Graph-partitioning based decomposition corresponds to one-dimensional (ID) decomposition (i.e., either rowwise or columnwise) through row/column permutations of the given matrix. We have recently proposed hypergraph-partitioning based decomposition schemes with better models for the communication requirement [4]. Both graph and hypergraph partitioning problems are NP-hard problems, and hence efficient heuristics are used for finding good decompositions. In graph/hypergraph approaches, both communication and load imbalance metrics are explicitly handled for minimization during the partitioning.

*This work is partially supported by the Commission of the European Communities, Directorate General for Industry under contract ITDC 204 82166 and The Scientific and Technical Research Council of Turkey under grant EEEAG-160
Graph/hypergraph partitioning based decomposition may not be appropriate for some applications. Reordering the matrix may not be feasible. Graph/hypergraph partitioning might be too expensive as a preprocessing step for the sake of parallelization. Finally, graph/hypergraph models may suffer from scalability in the decomposition of small matrices for large number of processors because of their $1 \mathrm{D}$ decomposition restriction.

In this work, we investigate the decomposition of sparse matrices without disturbing the given row/column ordering. In this approach, communication volume metric is handled implicitly by the selection of proper matrix partitioning and parallel SpMxV computation schemes at the beginning. Here, partitioning scheme refers to the scheme to be used for partitioning the given matrix to $K$ submatrices, where $K$ denotes the number of processors. Communication cost is determined by the partitioning scheme and the associated SpMxV algorithm. That is, the communication cost is assumed to be independent of the matrix sparsity pattern. Hence, load balance is the only metric explicitly considered in the decomposition. Cyclic (scattered) partitioning schemes automatically resolve the load balancing problem. However, these schemes suffer from high communication cost. Block partitioning schemes considerably reduce the communication cost in two-dimensional (2D) decomposition. Uniform block partitioning easily achieves perfect load balance in dense matrices. However, loadbalanced block partitioning becomes an important issue in the decomposition of irregularly sparse matrices.

We consider the load balancing problem in both $1 \mathrm{D}$ and 2D block partitioning schemes. 1D partitioning corresponds to rowwise or columnwise block striping [21]. Fig. 1(a) illustrates 4-way rowwise striping. 2D partitioning increases the scalability of the decomposition while reducing the volume of communication. Block-checkerboard partitioning [21] leads to an efficient SpMxV algorithm with low communication requirement [12]. This partitioning scheme is also referred to as rectilinear partitioning [25] and generalized block distribution (GBD) [23]. This scheme is very well suited to dense matrices and matrices with uniform sparsity pattern. However, it is hard to achieve good load balance on sparse matrices with non-uniform sparsity pattern because of the restriction of rectilinear splits on both rows and columns. Jagged rectilinear partitioning is commonly used to alleviate this problem. In this scheme, rectilinear splits are restricted to either rows or columns of the matrix thus increasing the search space for load balancing. In rowwise (columnwise) jagged partitioning, matrix is partitioned into $P$ horizontal (vertical) stripes, and every horizontal (vertical) stripe is independently partitioned into $Q$ submatrices, 


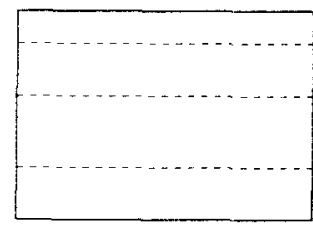

(a)

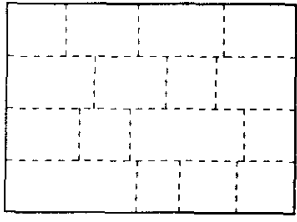

(b)
Figure 1: (a) 4-way rowwise striping and (b) $4 \times 4$-way rowwise jagged partitioning

where $K=P \times Q$. That is, splits span the entire matrix in one dimension, while they are jagged in the other dimension. This scheme is also referred to as semi-generalized block distribution (SBD) [23], basic partitioning configuration [5], and multiple recursive decomposition (MRD) [28]. Fig. 1(b) illustrates $4 \times 4$ rowwise jagged partitioning. Without loss of generality, we restrict our discussions to rowwise striped and jagged partitioning schemes. All results of this paper can easily be extended to columnwise schemes.

Despite the recent theoretical results on optimal block partitioning of workload arrays, heuristics are still commonly used in the sparse matrix community. This may be due to the ease of implementation, efficiency, and expectation of "good" quality decompositions. These heuristics are based on recursive decomposition (RD) of 1D workload arrays. For example, in rowwise striping, $K$-way decomposition is achieved through $\lg K$ bisection levels, where $K$ is a power of 2 . At each bisection step in a level, the current row stripe is divided evenly into two row stripes. Here, even division corresponds to two row stripes with equal number of nonzeros as much as possible. In jagged partitioning, this even bisection strategy is adopted both in the $P$-way row-striping and in the $Q$-way columnwise striping of every row stripe. Prime factorization of $K$ and $P, Q$ values is used to avoid the power-of-two restriction on these integer values for $1 \mathrm{D}$ and $2 \mathrm{D}$ decompositions, respectively [28].

Although optimal division can easily be achieved at every bisection step, the sequence of bisections may lead to poor load balancing. In Section 4, we demonstrate that qualities of the decompositions obtained through RD heuristic substantially deviate from those of the optimal ones through experimental results. In Sections 3.1 and 3.2, we propose efficient algorithms for optimal load-balancing in 1D striped and 2D jagged partitioning of sparse matrices. Experimental results presented in Section 4 demonstrate the feasibility of using optimal load balancing algorithms in sparse matrix domain. Proposed algorithms are 100 times faster than a single SpMxV computation, in the 64-way $1 \mathrm{D}$ decompositions, on the overall average. Proposed algorithms are also found to be much faster than random row/column permutation scheme which was proposed to produce probabilistically good decompositions [26]. Initial implementations of our jagged partitioning al gorithms are only $60 \%$ slower than a single $\mathrm{SpMxV}$ computation in the 64 -way $(8 \times 8) 2 \mathrm{D}$ decompositions, on the overall average. Proposed algorithms are also feasible in terms of memory requirement since they only need the conventional compressed storage scheme for the given matrix contrary to the existing optimal partitioning algorithms which depend on the existence of a dense workload matrix for 2D decomposition.

\section{Review on Partitioning of Workload Arrays}

ID partitioning of sparse matrices is equivalent to the chains-on-chains partitioning $(\mathrm{CCP})$ problem with unweighted edges. The objective of the CCP problem is to divide a $1 \mathrm{D}$ task array $T$ of length $M$ into $K$ consecutive parts such that the load of the maximally loaded part is minimized. In rowwise striping, $T[i]$ is equal to the number of nonzeros in row $i$ of the given $M \times N$ sparse matrix.

The first polynomial time algorithm for solving the CCP problem was proposed by Bokhari [2]. Bokhari's $O\left(M^{3} K\right)$ time algorithm is based on finding a minimum path on a layered graph. Nicol and O'Hallaron [24] reduced the complexity to $O\left(M^{2} K\right)$. The algorithm paradigms used in the following works can be classified as dynamic programming (DP), iterative refinement, and probe approaches. Anily and Federgruen [1] initiated the DP approach with an $O\left(M^{2} K\right)$ time algorithm. Hansen and Lih [11] independently proposed an $O\left(M^{2} K\right)$-time algorithm. Choi and Narahari [6], and Olstad and Manne [27] introduced asymptotically faster $O(M K)$-time, and $O((M-K) K)$-time DP-based algorithms, respectively. Iterative refinement approach starts with a partition and iteratively tries to improve the solution. The $O((M-K) K \lg K)$-time algorithm proposed by Manne and Sørevik [22] falls into this class.

The probe approach relies on repeated investigations for the existence of a partition with a bottleneck value no greater than a given value. Such a probing takes $\theta(M)$ time since every task has to be examined. Since probing needs to be performed repeatedly, an individual probe can efficiently be performed in $O(K \lg M)$-time through binary search, after performing an initial prefix-sum operation in $\theta(M)$-time for task chains with zero communication costs [14]. Later, $O(K \lg M)$-time probe algorithms were proposed to handle task chains with nonzero communication costs $[15,17,18$, $24]$. Finally, the complexity of an individual probe call was reduced to $O(K \lg (M / K))$ [10].

The probe approach goes back to Iqbal's [13, 17] work describing an $\epsilon$-approximate algorithm running in $O\left(K \lg M \lg \left(W_{t o t} / \epsilon\right)\right)$ time. Here, $W_{t o t}$ denotes the total task weight and $\epsilon$ denotes desired accuracy. Iqbal's algorithm exploits the observation that the bottleneck value is in the range $\left[W_{t o t} / K, W_{t o t}\right]$, and performs a binary search in this range by making $O\left(\lg \left(W_{\text {tot }} / \epsilon\right)\right)$ probe calls. This work was followed by several exact algorithms involving efficient schemes for the search over bottleneck values by considering only subchain weights. Nicol and O'Hallaron [24] proposed a search scheme which requires at most $4 M$ probe calls thus leading to a $O(M K \lg M)$-time algorithm. Iqbal and Bokhari [18] relaxed the restriction of this algorithm [24] on bounded task weight and communication cost, by proposing a condensation algorithm. Iqbal [16] and Nicol [24, 25] concurrently proposed an efficient search scheme which finds an optimal partition after only $O(K \lg M)$ probe calls, thus leading to an $O\left(M+(K \lg M)^{2}\right)$-time algorithm. Han et. al. [10] reduced the running time of this algorithm to $O\left(M+K^{2} \lg M \lg (M / K)\right)$ by exploiting their $O(K \lg (M / K))$-time probe function. Asymptotically best algorithms are optimal $O(M)$-time algorithm proposed by Frederickson [7], and $O\left(M+K^{1+\varepsilon}\right)$-time (for any small $\varepsilon>0$ ) algorithm proposed by Han et. al. [10]. However, these two works has mostly centered around decreasing the asymptotic running time, disregarding the run-time efficiency of the presented methods.

Theoretical work on optimal 2D partitioning is relatively rare. Nicol [25] conjectured the NP-completeness of the block checkerboard (2D rectilinear) partitioning problem by considering the closely related NP-complete multi-stage linear assignment problem [20]. The NP-completeness of 
this problem (GBD) has later been proven by Grigni and Manne [9]. Manne and Sørevik [23] extended the DP approach to optimal jagged partitioning of $2 \mathrm{D}$ workload arrays. Their algorithm runs in $O((M-P)(N-Q) P Q)$-time for jagged partitioning (SBD) of an $M \times N$ workload array to a $P \times Q$ processor array. In sparse matrix domain, the workload array $T$ represents the sparsity pattern of the given matrix $A$, such that $T[i, j]=0$ and $T[i, j]=1$ if $a_{i j}=0$ and $a_{i j}=1$, respectively.

\section{Proposed Load Balancing Algorithms}

The objective of this paper is to formulate both asymptotically and run-time efficient optimal load-balancing algorithms for $1 \mathrm{D}$ striped and $2 \mathrm{D}$ jagged partitioning schemes. An optimal decomposition corresponds to a partitioning which minimizes the number of nonzeros in the most heavily loaded processor (bottleneck processor). The load of the bottleneck processor is called the bottleneck value of the partition. Efficiency in terms of memory requirement is also considered in these formulations since maintaining an $M \times . V$ workload array for an $M \times N$ sparse matrix is not acceptable. So, our algorithms use either the row compressed storage (RCS) or column compressed storage (CCS) schemes for the given sparse matrix. RCS is used for rowwise striped and rowwise jagged partitioning schemes.

We have developed and experimented several optimal load balancing algorithms. In this section, we present and discuss only two algorithms for ID striped and 2D jagged partitioning schemes due to the lack of space. These algorithms seem to be the most promising algorithms according to the current implementations. We restrict our discussion to probe-based approaches because of extremely high execution times of DP-based approaches on sparse test matrices. This finding is experimentally verified in Section 4 .

\subsection{One-Dimensional Striped Partitioning}

In this section, we consider optimal $\mathrm{K}$-way row striping of an $H \times N$ sparse matrix.

\subsubsection{Binary Search Algorithm}

Iqbal's [17] binary search method is a very promising approach for sparse matrix decomposition for the following two reasons. First, tight bounds can be set for the bottleneck value of an optimal solution. The bottleneck value $B_{\text {opt }}$ of an optimal partition ranges between $L B=B^{*}$ and $\mathrm{UB}=B^{\star}+w_{\max }$, where $w_{\max }$ is the maximum element in the workload array, and $B^{*}=W_{\text {tot }} / K$ is the ideal bottle neck value. In rowwise striping, $W_{\text {tot }}=Z$ corresponds to the total number of nonzeros in the sparse matrix, and $w_{\max }$ is the number of nonzeros in the most dense row. Note that $w_{\text {max }} \ll N$ in most sparse matrices arising in various fields. Second, the $\epsilon$-approximation restriction does not apply since the workload array is composed of integers.

The binary search algorithm is illustrated in Fig. 2. The workload array $T$ is such that $T[i]$ is equal to the number of nonzeros in the $i$ th row $r_{i}$ of the matrix, i.e., $T[i]=w_{i}$. Prefix sum on the task array $T$ enables the constant-time computation of the subchain weight $W_{i, j}=\sum_{k=i}^{j} w_{k}$ of the rowstripe $r_{i}, r_{i+1}, \ldots, r_{j}$ through $W_{i, j}=T[j]-T[i-1]$. Integer weights in the task array restrict the optimal bottleneck value $B_{\text {opt }}$ to $U B-L B=w_{\max }$ distinct integer values within the range $L B \leq B_{\text {opt }}<U B$. Hence, binary search can be efficiently used to find $B_{\text {opt }}$ through probes in the interval $[L B, U B]$.

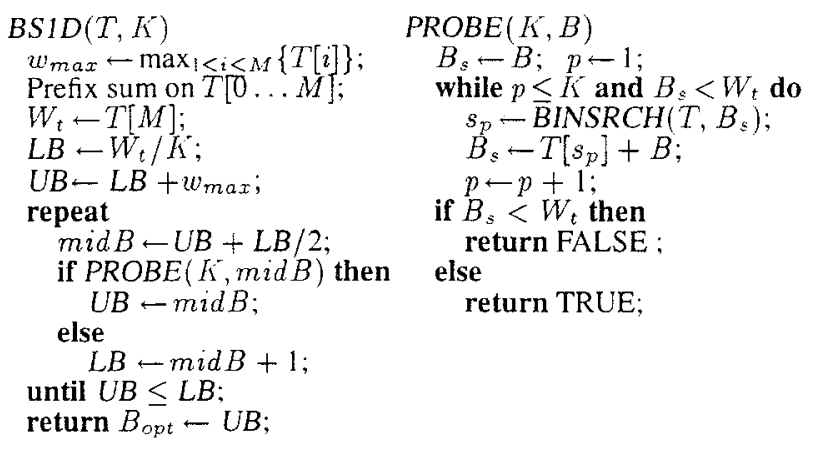

Figure 2: Binary search algorithm for $1 \mathrm{D} K$-way rowwise striping.

Given a bottleneck value $B, \operatorname{PROBE}(T, K, B)$ tries to find a $K$-way partition of $T$ with a bottleneck value no greater than $B$. PROBE finds the largest index $s_{1}$ so that $W_{1, s_{1}} \leq B$, and assigns the row-stripe $r_{1}, r_{2}, \ldots r_{s_{1}}$ to processor 1 . Hence, the first row in the second processor is $r_{s_{1}+1}$. PROBE then similarly finds the largest index $s_{2}$ so that $W_{s_{1}+1, s_{2}} \leq B$, and assigns the row-stripe $r_{s_{1}+1}, r_{s_{1}+2}, \ldots r_{s_{2}}$ to processor 2 . This process continues until either all rows are assigned or the processors are exhausted. The former case denotes the existence of a partition with bottleneck value no greater than $B$, whereas the latter shows the inexistence of a partition with bottleneck value smaller than or equal to $B$. As seen in Fig 2 , the indices $s_{1}, s_{2}, \ldots s_{K-1}$ are efficiently found through binary search (BINSRCH) on the prefix-summed array $T$. Note that an optimal solution can easily be constructed by making a last PROBE call with $B_{\text {opt }}$.

The complexity of one PROBE call is $O(K \lg M)$. The binary search algorithm makes $\lg w_{\max }$ PROBE calls. Thus, the overall complexity of the algorithm is $O\left(M+K \lg M \lg w_{\max }\right)$ together with the initial $O(M)$ time prefix-sum operation. The algorithm is surprisingly fast, so that the initial prefix-sum operation dominates the overall execution time. Fortunately, the data structure for the RCS scheme is efficiently exploited to avoid the initial prefix-sum operation without any additional operations, thus reducing the complexity to $O\left(K \lg M \lg w_{\max }\right)$.

In this work, we further exploit the nice bounds on optimal bottleneck value to restrict the search space for $s_{p}$ separator values during BINSRCH in PROBE calls. That is, for each processor $p=1,2, \ldots K-1, S L_{p} \leq s_{p} \leq S H_{p}$, where $S L_{p}$ and $S H_{p}$ correspond to the smallest and largest indices such that $W_{1, S L_{p}} \geq p\left(B^{*}-w_{\max }(K-p) / K\right)$ and $W_{1, S H_{p}} \leq p\left(B^{*}+w_{\max }(K-p / K)\right)$, respectively. This scheme reduces the complexity of an individual probe call to $O\left(K \lg K+K \lg \left(w_{\max } / w_{\text {avg }}\right)\right)$, where $w_{\text {avg }}=W_{\text {tot }} / M$ denotes the average number of nonzeros per row. This reduces the overall complexity to $O\left(K \lg w_{\max } \lg K+\right.$ $\left.K \lg w_{\max } \lg \left(w_{\max } / w_{\text {avg }}\right)+K \lg M\right)$, together with the initial cost of $O(K \lg M)$ for setting the $S L_{p}$ and $S H_{p}$ values.

\subsubsection{Bidding Algorithm}

In this work, we propose an iterative-refinement scheme which is much more effective than the one proposed by Manne and Sørevik [22]. The proposed algorithm, namely the BIDDING algorithm, is presented in Fig. 3. In this algorithm, we dynamically increase the bottleneck value 


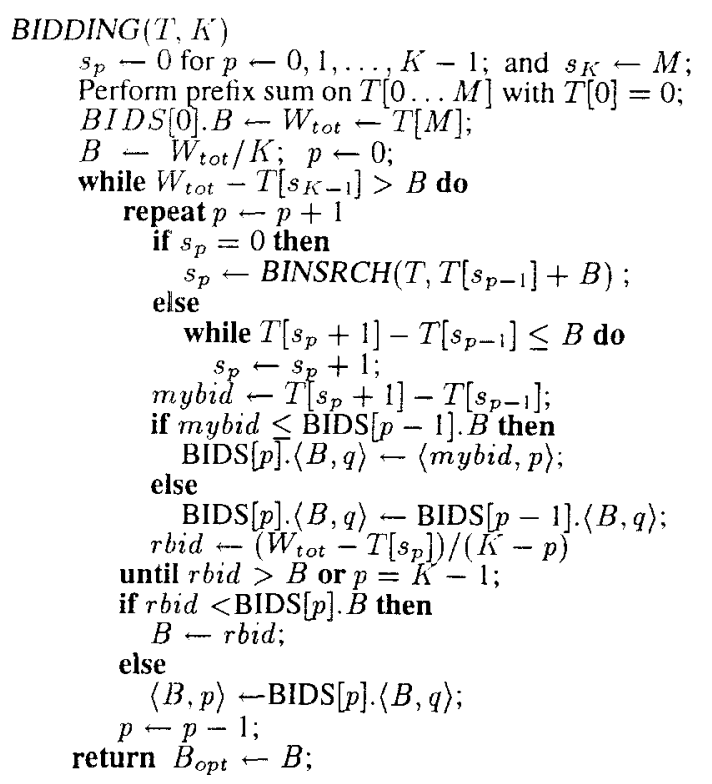

Figure 3: Bidding algorithm for $1 \mathrm{D} K$-way row striping.

$B$, starting from the perfect bottleneck value $B^{*}$, until a feasible partition is obtained. This leads to an incremental probing scheme, where the decision is given by modifying the separators from the previous probe call. The separator indices $s_{1}, s_{2}, \ldots, s_{K-1}$ are set as the largest indices such that $W_{s_{p-1}+1, s_{p}} \leq B$ with $s_{0}=0$ for $p=1,2, \ldots, K-1$. As in the conventional probing scheme, $W_{s_{K-1}, M}>B$ denotes the infeasibility of the current $B$ value. After detecting an infeasible $B$ value, the important issue is to determine the next larger $B$ value to be investigated. Undoubtfully, at least one of the separators should move to the right for a feasible partition. So, the next larger $B$ value is computed by selecting the minimum of the set of $\left\{W_{s_{p-1}+1, s_{p}+1}\right\}_{p=1}^{K-1} \cup$ $\left\{W_{s_{k-1}+1, M}\right\}$ values. We call the $W_{s_{p-1}+1, s_{p}+1}$ value the bid of processor $p$, which refers to the load of processor $p$ if the first row $r_{s_{p}+1}$ of the next processor is added to processor. Note that the bid of the last processor $K$ is equal to the load of the remaining rows. If the best bid $B$ comes from part $p^{*}$, probing with new $B$ is performed only for the remaining processors $\left(p^{*}, p^{*}+1, \ldots\right)$. In this scheme, we prefer to determine the new positions of the separators by moving them to the right one by one, since their new positions are likely to be in a close neighborhood of their previous values. Note that binary search is used only for setting the separator indices for the first time. As seen in Fig. 3, we maintain prefix-minimum array BIDS for computing the next larger $B$ value in constant time. Here, BIDS is an array of records of length $K$, where $B I D S[p] . B$ and $B I D S[p] . q$ store the best bid value of the first $p$ processors and the corresponding processor, respectively. BIDS[0] is used to enable the running prefix-minimum operation.

After the separator index $s_{p}$ is set for processor $p$, the repeat-until-loop terminates if it is not possible to partition the remaining segment $T_{s_{p}+1, M}$ into $K-p$ processors without exceeding the current $B$ value, i.e., rbid $=$ $\left(W_{t o t}-T\left[s_{p}\right]\right) /(K-p)>B$. In this case, the next larger $B$ value is determined by considering the best bid among the first $p$ processors and rbid. Here, rbid represents the bottleneck value of the perfect $(K-p)$-way partitioning of the remaining segment $T_{s_{p}+1, M}$. Note that this variable also stores the bid of the last processor, when all separator indices $s_{1}, s_{2}, \ldots, s_{K-1}$ are assigned nonzero values.

\subsection{Two-Dimensional Jagged Partitioning}

In this section, we consider optimal $(P \times Q)$-way rowwise jagged partitioning of an $M \times N$ sparse matrix. Binary search method can be extended to $2 \mathrm{D}$ jagged partitioning by setting tight bounds on the value of the optimal bottleneck value and defining an appropriate $2 \mathrm{D}$ probe function. We compute the bounds by constructing a conditionally optimal jagged partition as follows. We first construct an optimal ID $P$-way rowwise striping of the given matrix. Then, we construct a jagged partition by constructing optimal 1D columnwise striping of every row stripe found in the first phase. The upper bound $U B$ is set to the bottleneck value of this conditionally optimal jagged partition. The lower bound $L B$ is computed by dividing the bottleneck value of the optimal rowwise striping by $Q$. The bounds on $L B$ and $U B$ can be derived as $L B \geq Z / P Q$ and $U B \leq Z / P Q+w_{\max } / P+w_{c \max }$, respectively. Hence, the search range for binary search will always be less than $w_{\text {rmax }} / P+w_{\text {cmax }}$ distinct integers. Here, $w_{\text {rmax }}$ and $w_{\text {cmax }}$ denote the number of nonzeros in the most dense row and column, respectively.

In this work, we propose a $2 \mathrm{D}$ probe algorithm. Given a bottleneck value $B, P R O B E 2 D$ tries to find a $P \times Q$-way jagged partition of matrix $A$ with a bottleneck value no greater than $B$. PROBE2D finds the largest row index $R_{1}$ so that there exists a $Q$-way columnwise striping of the the rowstripe (submatrix) $A_{1, R_{1}}$ with a bottleneck value no greater than $B$. PROBE2D then similarly finds the largest row index $R_{2}$ so that there exists a $Q$-way columnwise striping of the next row-stripe $A_{R_{1}+1, R_{2}}$ with a bottleneck value no greater than $B$. This process continues until either all rows are consumed, or $P$ row-stripes are obtained. The former case denotes the existence of a jagged partition with bottleneck value no greater than $B$, whereas the latter shows the inexistence of a partition with bottleneck value smaller than or equal to $B$. In a similar way to our $B S 1 D$ algorithm, we further exploit the nice upper bound on optimal bottleneck value to restrict the search space for $R_{p}$ separator values during the binary search in PROBE2D calls. That is, for each processor $p=1,2, \ldots K-1, R L_{p} \leq R_{p} \leq R H_{p}$, where $R L_{p}$ and $R H_{p}$ correspond to the smallest and largest row indices such that $W_{1, R L_{p}} \geq U B \times Q \times p$ and $W_{1, R H_{p}} \leq W_{\text {tot }}-U B \times Q \times p$, respectively. As seen in Fig. 4, we favor a different 1D probing scheme (PROBE1D) which does not adopt prefixsum, since the same row-stripe is not likely to be explored multiple times for $Q$-way ID probing.

1D bidding algorithm presented in Section 3.1.2 is also extended to $2 \mathrm{D}$ jagged partitioning. The critical point in this algorithm is how to compute the next larger $B$ value. In 2D bidding algorithm, $P$ row-stripes bid for the next $B$ value. The bid of each row-stripe is determined by the optimal bottleneck value of columnwise striping of the submatrix composed of the current rows of the stripe and the first row of the next stripe. Due to lack of space, we cannot present the details here.

\section{Experimental Results}

We have experimented the performance of the proposed load balancing algorithms for the rowwise striped and jagged partitioning of various test matrices arising in linear programming domain. Table 1 illustrates the prop- 

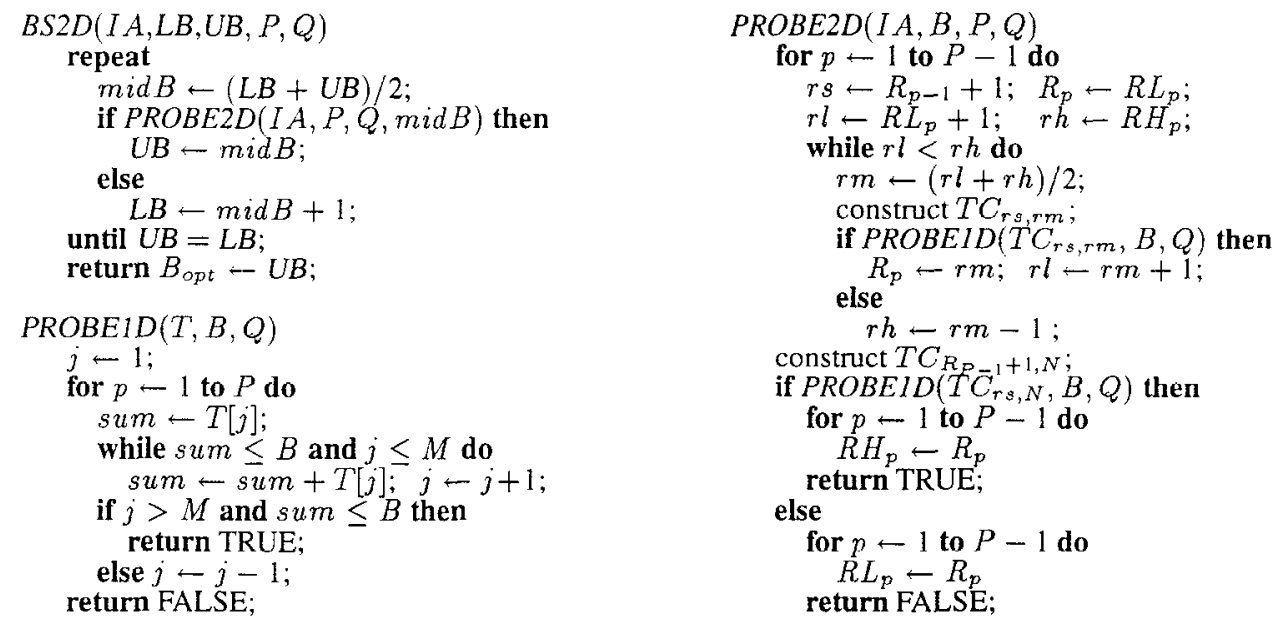

Figure 4: Binary search algorithm for $2 \mathrm{D} P \times Q$-way jagged partitioning

Table 1: Properties of sparse test matrices.

\begin{tabular}{|c|c|c|c|c|c|c|}
\hline \multirow{3}{*}{ name } & \multirow{3}{*}{$\begin{array}{c}\text { number } \\
\text { of } \\
\text { row/col }\end{array}$} & \multicolumn{4}{|c|}{ number of non-zeros } & \multirow{3}{*}{$\begin{array}{l}\text { ex. time } \\
\text { SpMxV } \\
\text { msecs }\end{array}$} \\
\hline & & \multirow[t]{2}{*}{ total } & \multicolumn{3}{|c|}{ рег row/column } & \\
\hline & & & avg & $\min$ & $\max$ & \\
\hline pilot87 & 2030 & 238624 & 117.55 & 1 & 738 & 43.90 \\
\hline cre-b & 9648 & 398806 & 41.34 & 1 & 904 & 77.80 \\
\hline cre $\sim d$ & 8926 & 372266 & 41.71 & $\mathrm{~T}$ & 845 & 72.20 \\
\hline ken-11 & 14694 & 82454 & 5.61 & 2 & 243 & 19.65 \\
\hline ken- 18 & $105 ! 27$ & 609271 & 5.80 & 2 & 649 & 167.40 \\
\hline $\mathrm{CO9}$ & 10789 & 249205 & 23.10 & $\overline{\mathrm{I}}$ & 707 & 51.45 \\
\hline CQ9 & 9278 & 221590 & 23.88 & I & 702 & 45.90 \\
\hline $\mathrm{NL}$ & 7039 & 105089 & 14.93 & $\overline{1}$ & 361 & 22.55 \\
\hline $\bmod 2$ & 34774 & 604910 & 17.40 & 1 & 941 & 124.05 \\
\hline world & 34506 & 582064 & 16.87 & 1 & 972 & 119.45 \\
\hline
\end{tabular}

erties of the test matrices. These test matrices are obtained from Netlib suite [8], and IOWA Optimization Center (ftp:/col.biz.uiowa.edu:pub/testprob/lp/gondzio/). The sparsity pattern of these matrices are obtained by multiplying the respective rectangular constraint matrices with their tranposes. Table $\mathrm{I}$ also displays the execution time of a single SpMxV operation for each test matrix.

All algorithms are implemented in $C$ language. All experiments are carried out on a workstation equipped with a $133 \mathrm{MHz}$ PowerPC with $512-\mathrm{KB}$ external cache, and $64 \mathrm{MB}$ of memory. We have experimented $16,32,64,128,256$ way row-striping and $4 \times 4,4 \times 8,8 \times 8,8 \times 16,16 \times 16$ way jagged partitioning of every test matrix.

Table 2 illustrates relative performance results of various load balancing algorithms. In this table, $\mathrm{RD}$ refers to the recursive decomposition heuristic mentioned in Section 1. Recall that $\mathrm{RD}$ is equaivalent to MRD scheme mentioned earlier in Section 1. RD scheme is implemented as efficiently as possible by adopting binary search on $1 \mathrm{D}$ prefixsummed workload arrays. DP and Nic. refer to the dynamic programming and Nicol's probe-based 1D decomposition schemes, implemented with respect to guidelines provided in $[6,27]$ and $[25]$, respectively. BS and Bid stand for the proposed binary search and bidding algorithms described in Section 3.

In Table 2, percent load imbalance values are computed as $\left(W_{\max }-B^{*}\right) / B^{*}$, where $W_{\max }$ denotes the number of nonzeros in the bottleneck processor (part, submatrix), and $B^{*}=Z / K$ denotes the number of nonzeros in every processor under perfectly balanced partitioning. OPT denotes the percent load imbalance obtained by the exact algorithms. The table clearly shows that considerably better decompositions can be obtained by using optimal load balancing algorithms instead of heuristics. The quality gap between the solutions of exact algorithms and heuristics increases with decreasing granularity, as expected. As also expected, 2D jagged partitioning always produces better decompositions than 1D striping. This quality gap becomes considerably large for larger number of processors.

Table 2 also displays the execution times of various decomposition algorithms normalized with respect to a single SpMxV time. Note that normalized execution times of $1 D$ decomposition algorithms are multiplied by 100 because of the difficulty of displaying extremely low execution times of the proposed binary search (BS), and bidding (Bid) algorithms. DP approaches are not recommended for sparse matrix decomposition because of their considerably large execution times relative to those of the proposed algorithms. In $1 \mathrm{D}$ decompostion of sparse matrices, both of our algorithms are definitely faster than Nicol's algorithm. Although our algorithms are slower than RD heuristic, their additional processing time is justified because of their considerably better decompositon quality and extremely low execution times compared to a single SpMxV computation time.

The execution times for 2D partitioning algorithms are relatively high compared to $1 \mathrm{D}$ partitioning, however the quality of the partitions and the execution times of the initial implementations encourage further research for faster algorithms and implementations.

\section{Conclusion and Future Research}

Efficient optimal load balancing algorithms were proposed for 1D striped and 2D jagged partitioning of sparse matrices. Experimental results on a wide set of test matrices verified that considerably better decompositions can be obtained by using optimal load balancing algorithms instead of heuristics. The proposed algorithms were found to be orders of magnitude faster than a single matrix-vector multiplication in $1 \mathrm{D}$ decomposition. The proposed algorithms for 2D partitioning are slightly slower than a matrix-vector multiplication, while producing significiantly better decompositions than the heuristics. We are currently working on improving the speed performance of our 2D load balancing algorithms. 
Table 2: Relative performance results of various load balancing algorithms.

\begin{tabular}{|c|c|c|c|c|c|c|c|c|c|c|c|c|c|c|}
\hline \multirow{3}{*}{ nalne } & \multicolumn{8}{|c|}{ ID Decompostion (rowwise striping) } & \multicolumn{6}{|c|}{ 2D Decomposition (rowwise jagged) } \\
\hline & \multirow[t]{2}{*}{$\mathrm{K}$} & \multicolumn{2}{|c|}{$\begin{array}{l}\text { percent load } \\
\text { imbalance }\end{array}$} & \multicolumn{5}{|c|}{$\begin{array}{l}\text { T00 } \text { execution uime } \\
\text { normalized wrt SpMxV }\end{array}$} & \multirow{2}{*}{$\begin{array}{c}\mathrm{K} \\
\mathrm{PxQ} \\
\end{array}$} & \multicolumn{2}{|c|}{$\begin{array}{l}\text { percent load } \\
\text { imbalance }\end{array}$} & \multicolumn{3}{|c|}{$\begin{array}{c}\text { execution time } \\
\text { norm. wrt SpMxV }\end{array}$} \\
\hline & & OPT & RD & RD & DP & Nic. & BS & Bid & & OPT & RD & RD & Bid & BS \\
\hline pilot87 & $\begin{array}{r}16 \\
32 \\
64 \\
128 \\
256 \\
\end{array}$ & $\begin{array}{r}1.15 \\
1.85 \\
2.99 \\
6.05 \\
14.26\end{array}$ & $\begin{array}{r}1.15 \\
4.39 \\
5.89 \\
12.22 \\
36.14\end{array}$ & $\begin{array}{l}0.05 \\
0.07 \\
0.11 \\
0.25 \\
0.43\end{array}$ & $\begin{array}{r}12 \\
25 \\
50 \\
96 \\
174\end{array}$ & $\begin{array}{r}4 \\
13 \\
44 \\
148 \\
495\end{array}$ & $\begin{array}{l}0.34 \\
0.46 \\
1.03 \\
1.94 \\
3.64\end{array}$ & $\begin{array}{l}0.06 \\
0.23 \\
0.46 \\
1.20 \\
4.50\end{array}$ & $\begin{array}{r}4 \times 4 \\
4 \times 8 \\
8 \times 8 \\
8 \times 16 \\
16 \times 16\end{array}$ & $\begin{array}{l}0.46 \\
0.66 \\
0.98 \\
1.76 \\
2.13\end{array}$ & $\begin{array}{l}0.5 \overline{2} \\
1.02 \\
1.27 \\
2.78 \\
4.38\end{array}$ & $\begin{array}{l}0.46 \\
0.46 \\
0.46 \\
0.47 \\
0.48\end{array}$ & $\begin{array}{l}0.54 \\
0.51 \\
0.64 \\
0.76 \\
0.97\end{array}$ & $\begin{array}{l}0.50 \\
0.48 \\
0.56 \\
0.67 \\
0.77\end{array}$ \\
\hline cre-b & $\begin{array}{r}16 \\
32 \\
64 \\
128 \\
256 \\
\end{array}$ & $\begin{array}{r}0.25 \\
0.81 \\
1.20 \\
2.51 \\
10.02\end{array}$ & $\begin{array}{r}0.84 \\
3.92 \\
6.12 \\
17.05 \\
20.74\end{array}$ & $\begin{array}{l}0.03 \\
0.05 \\
0.09 \\
0.18 \\
0.37\end{array}$ & $\begin{array}{r}33 \\
66 \\
135 \\
275 \\
533\end{array}$ & $\begin{array}{r}4 \\
16 \\
61 \\
214 \\
671\end{array}$ & $\begin{array}{l}0.64 \\
0.84 \\
1.61 \\
2.44 \\
3.53\end{array}$ & $\begin{array}{l}0.06 \\
0.13 \\
0.22 \\
0.80 \\
1.99\end{array}$ & $\begin{array}{r}4 \times 4 \\
4 \times 8 \\
8 \times 8 \\
8 \times 16 \\
16 \times 16\end{array}$ & $\begin{array}{l}0.10 \\
0.30 \\
0.49 \\
0.88 \\
1.42\end{array}$ & $\begin{array}{l}0.21 \\
1.23 \\
1.92 \\
3.22 \\
5.92\end{array}$ & $\begin{array}{l}0.49 \\
0.50 \\
0.53 \\
0.54 \\
0.61\end{array}$ & $\begin{array}{l}0.67 \\
1.38 \\
1.58 \\
2.08 \\
3.58\end{array}$ & $\begin{array}{l}0.60 \\
0.69 \\
0.86 \\
1.18 \\
1.84\end{array}$ \\
\hline cre-d & $\begin{array}{r}16 \\
32 \\
64 \\
128 \\
256 \\
\end{array}$ & $\begin{array}{r}0.45 \\
0.63 \\
1.73 \\
2.88 \\
10.85\end{array}$ & $\begin{array}{r}0.53 \\
3.74 \\
4.34 \\
16.70 \\
35.20\end{array}$ & $\begin{array}{l}0.03 \\
0.06 \\
0.10 \\
0.19 \\
0.37\end{array}$ & $\begin{array}{r}32 \\
70 \\
137 \\
274 \\
532 \\
\end{array}$ & $\begin{array}{r}4 \\
18 \\
62 \\
218 \\
677\end{array}$ & $\begin{array}{l}0.55 \\
0.83 \\
1.52 \\
2.63 \\
3.88\end{array}$ & $\begin{array}{l}0.03 \\
0.10 \\
0.28 \\
0.80 \\
1.63\end{array}$ & $\begin{array}{r}4 \times 4 \\
4 \times 8 \\
8 \times 8 \\
8 \times 16 \\
16 \times 16\end{array}$ & $\begin{array}{l}0.14 \\
0.25 \\
0.64 \\
1.09 \\
1.57\end{array}$ & $\begin{array}{l}0.38 \\
0.61 \\
1.98 \\
7.00 \\
6.25\end{array}$ & $\begin{array}{l}0.50 \\
0.51 \\
0.53 \\
0.53 \\
0.60\end{array}$ & $\begin{array}{l}0.62 \\
0.82 \\
1.16 \\
1.86 \\
3.32\end{array}$ & $\begin{array}{l}0.60 \\
0.62 \\
0.99 \\
1.16 \\
2.05\end{array}$ \\
\hline ken-11 & $\begin{array}{r}16 \\
32 \\
64 \\
128 \\
256 \\
\end{array}$ & $\begin{array}{l}0.21 \\
1.18 \\
1.29 \\
6.80 \\
7.11 \\
\end{array}$ & $\begin{array}{r}0.98 \\
3.74 \\
13.77 \\
13.17 \\
50.89\end{array}$ & $\begin{array}{l}0.10 \\
0.15 \\
0.31 \\
0.76 \\
1.53\end{array}$ & $\begin{array}{r}227 \\
460 \\
930 \\
1859 \\
3618 \\
\end{array}$ & $\begin{array}{r}19 \\
77 \\
292 \\
1011 \\
3360\end{array}$ & $\begin{array}{r}3.56 \\
4.83 \\
6.62 \\
10.43 \\
16.03\end{array}$ & $\begin{array}{r}0.25 \\
1.91 \\
2.67 \\
20.36 \\
36.01\end{array}$ & $\begin{array}{r}4 \times 4 \\
4 \times 8 \\
8 \times 8 \\
8 \times 16 \\
16 \times 16\end{array}$ & $\begin{array}{l}0.07 \\
0.13 \\
0.36 \\
0.59 \\
1.22\end{array}$ & $\begin{array}{l}0.11 \\
0.13 \\
1.14 \\
1.21 \\
2.46\end{array}$ & $\begin{array}{l}0.66 \\
0.68 \\
0.88 \\
0.96 \\
1.24\end{array}$ & $\begin{array}{r}1.83 \\
2.60 \\
6.11 \\
10.28 \\
27.30\end{array}$ & $\begin{array}{l}1.40 \\
1.50 \\
3.28 \\
2.75 \\
4.99\end{array}$ \\
\hline ken- 18 & $\begin{array}{r}16 \\
32 \\
64 \\
128 \\
256\end{array}$ & $\begin{array}{l}0.17 \\
0.23 \\
0.89 \\
0.95 \\
4.58\end{array}$ & $\begin{array}{l}0.56 \\
2.26 \\
2.26 \\
9.08 \\
9.08\end{array}$ & $\begin{array}{l}0.01 \\
0.02 \\
0.06 \\
0.13 \\
0.26\end{array}$ & $\begin{array}{r}243 \\
499 \\
996 \\
1950 \\
3822\end{array}$ & $\begin{array}{r}5 \\
19 \\
73 \\
269 \\
980\end{array}$ & $\begin{array}{l}3.05 \\
3.76 \\
4.24 \\
5.23 \\
7.35\end{array}$ & $\begin{array}{r}0.18 \\
0.27 \\
1.82 \\
2.82 \\
27.63\end{array}$ & $\begin{array}{r}4 \times 4 \\
4 \times 8 \\
8 \times 8 \\
8 \times 16 \\
16 \times 16\end{array}$ & $\begin{array}{l}1.2 \\
0.00 \\
0.08 \\
0.15 \\
0.21 \\
0.42\end{array}$ & $\begin{array}{l}0.70 \\
0.01 \\
0.38 \\
0.59 \\
1.37 \\
1.72\end{array}$ & $\begin{array}{l}0.80 \\
0.81 \\
0.99 \\
0.99 \\
1.36\end{array}$ & $\begin{array}{r}1.78 \\
9.55 \\
20.74 \\
23.67 \\
60.82\end{array}$ & $\begin{array}{l}1.77 \\
3.06 \\
4.51 \\
3.99 \\
8.91\end{array}$ \\
\hline $\mathrm{CO} 9$ & $\begin{array}{r}16 \\
32 \\
64 \\
128 \\
256 \\
\end{array}$ & $\begin{array}{r}0.34 \\
1.07 \\
1.93 \\
4.73 \\
13.62\end{array}$ & $\begin{array}{r}1.37 \\
4.60 \\
4.96 \\
18.75 \\
42.58\end{array}$ & $\begin{array}{l}0.02 \\
0.06 \\
0.12 \\
0.25 \\
0.52\end{array}$ & $\begin{array}{r}63 \\
126 \\
254 \\
533 \\
1013\end{array}$ & $\begin{array}{r}6 \\
24 \\
94 \\
328 \\
1062\end{array}$ & $\begin{array}{l}0.97 \\
1.55 \\
2.53 \\
4.47 \\
5.54\end{array}$ & $\begin{array}{r}0.10 \\
0.39 \\
1.21 \\
3.98 \\
19.44\end{array}$ & $\begin{array}{r}4 \times 4 \\
4 \times 8 \\
8 \times 8 \\
8 \times 16 \\
16 \times 16\end{array}$ & $\begin{array}{l}0.36 \\
0.45 \\
1.08 \\
1.39 \\
1.90\end{array}$ & $\begin{array}{r}0.40 \\
1.48 \\
1.62 \\
6.12 \\
13.20\end{array}$ & $\begin{array}{l}0.52 \\
0.53 \\
0.58 \\
0.59 \\
0.62\end{array}$ & $\begin{array}{l}1.31 \\
1.51 \\
3.40 \\
5.15 \\
9.40\end{array}$ & $\begin{array}{l}0.84 \\
0.86 \\
1.40 \\
1.75 \\
3.96\end{array}$ \\
\hline $\mathrm{CQ9}$ & $\begin{array}{r}16 \\
32 \\
64 \\
128 \\
256 \\
\end{array}$ & $\begin{array}{r}0.58 \\
0.80 \\
1.43 \\
3.51 \\
14.72 \\
\end{array}$ & $\begin{array}{r}0.58 \\
2.24 \\
7.64 \\
22.34 \\
58.62 \\
\end{array}$ & $\begin{array}{l}0.04 \\
0.07 \\
0.13 \\
0.33 \\
0.59\end{array}$ & $\begin{array}{r}55 \\
118 \\
233 \\
485 \\
908\end{array}$ & $\begin{array}{r}7 \\
26 \\
95 \\
336 \\
1104\end{array}$ & $\begin{array}{l}0.98 \\
1.42 \\
2.40 \\
3.70 \\
6.54\end{array}$ & $\begin{array}{r}0.22 \\
0.22 \\
0.87 \\
3.05 \\
17.65\end{array}$ & $\begin{array}{r}4 \times 4 \\
4 \times 8 \\
8 \times 8 \\
8 \times 16 \\
16 \times 16\end{array}$ & $\begin{array}{l}0.28 \\
0.48 \\
0.86 \\
1.38 \\
2.36\end{array}$ & $\begin{array}{r}0.66 \\
1.84 \\
1.84 \\
4.44 \\
13.00\end{array}$ & $\begin{array}{l}0.50 \\
0.51 \\
0.56 \\
0.57 \\
0.66\end{array}$ & $\begin{array}{l}1.07 \\
2.00 \\
2.39 \\
4.11 \\
8.68\end{array}$ & $\begin{array}{l}0.73 \\
0.77 \\
1.13 \\
\mathbf{1 . 8 1} \\
3.44\end{array}$ \\
\hline NL & $\begin{array}{r}16 \\
32 \\
64 \\
128 \\
256 \\
\end{array}$ & $\begin{array}{r}0.35 \\
0.85 \\
2.37 \\
4.99 \\
14.25 \\
\end{array}$ & $\begin{array}{r}1.20 \\
3.44 \\
5.60 \\
22.78 \\
60.78 \\
\end{array}$ & $\begin{array}{l}0.04 \\
0.13 \\
0.31 \\
0.58 \\
1.15 \\
\end{array}$ & $\begin{array}{r}89 \\
173 \\
393 \\
717 \\
1419 \\
\end{array}$ & $\begin{array}{r}12 \\
44 \\
167 \\
597 \\
1714 \\
\end{array}$ & $\begin{array}{r}2.00 \\
2.44 \\
4.21 \\
5.99 \\
11.31\end{array}$ & $\begin{array}{r}0.22 \\
0.55 \\
2.11 \\
5.21 \\
23.61 \\
\end{array}$ & $\begin{array}{r}4 \times 4 \\
4 \times 8 \\
8 \times 8 \\
8 \times 16 \\
16 \times 16 \\
\end{array}$ & $\begin{array}{l}0.26 \\
0.46 \\
0.79 \\
1.34 \\
1.83 \\
\end{array}$ & $\begin{array}{l}0.49 \\
1.89 \\
2.86 \\
5.84 \\
5.48 \\
\end{array}$ & $\begin{array}{l}0.55 \\
0.55 \\
0.61 \\
0.63 \\
0.75 \\
\end{array}$ & $\begin{array}{r}1.51 \\
1.88 \\
3.48 \\
4.41 \\
10.13 \\
\end{array}$ & $\begin{array}{l}0.93 \\
0.89 \\
1.71 \\
1.86 \\
4.52 \\
\end{array}$ \\
\hline $\bmod 2$ & $\begin{array}{r}16 \\
32 \\
64 \\
128 \\
256 \\
\end{array}$ & $\begin{array}{l}0.03 \\
0.07 \\
0.18 \\
0.41 \\
1.23\end{array}$ & $\begin{array}{r}0.06 \\
0.19 \\
2.18 \\
2.46 \\
18.92\end{array}$ & $\begin{array}{l}0.02 \\
0.04 \\
0.08 \\
0.18 \\
0.34\end{array}$ & $\begin{array}{r}92 \\
185 \\
374 \\
746 \\
1500\end{array}$ & $\begin{array}{r}5 \\
20 \\
73 \\
275 \\
959 \\
\end{array}$ & $\begin{array}{l}1.41 \\
1.65 \\
2.14 \\
3.35 \\
5.24\end{array}$ & $\begin{array}{l}0.02 \\
0.08 \\
0.24 \\
0.58 \\
3.69 \\
\end{array}$ & $\begin{array}{r}4 \times 4 \\
4 \times 8 \\
8 \times 8 \\
8 \times 16 \\
16 \times 16\end{array}$ & $\begin{array}{l}0.05 \\
0.09 \\
0.11 \\
0.28 \\
0.43\end{array}$ & $\begin{array}{l}0.11 \\
0.13 \\
0.20 \\
1.84 \\
2.92 \\
\end{array}$ & $\begin{array}{l}0.63 \\
0.64 \\
0.64 \\
0.65 \\
0.80 \\
\end{array}$ & $\begin{array}{r}1.80 \\
2.12 \\
3.57 \\
5.20 \\
14.05 \\
\end{array}$ & $\begin{array}{l}1.21 \\
1.06 \\
1.59 \\
2.14 \\
4.09 \\
\end{array}$ \\
\hline world & $\begin{array}{r}16 \\
32 \\
64 \\
128 \\
256\end{array}$ & $\begin{array}{l}0.04 \\
0.08 \\
0.28 \\
0.76 \\
1.11\end{array}$ & $\begin{array}{r}0.09 \\
0.27 \\
4.73 \\
6.37 \\
27.41\end{array}$ & $\begin{array}{l}0.02 \\
0.03 \\
0.09 \\
0.18 \\
0.36\end{array}$ & $\begin{array}{r}92 \\
192 \\
376 \\
762 \\
1468\end{array}$ & $\begin{array}{r}5 \\
20 \\
78 \\
282 \\
1023\end{array}$ & $\begin{array}{l}1.34 \\
1.72 \\
2.26 \\
3.43 \\
5.40\end{array}$ & $\begin{array}{l}0.04 \\
0.08 \\
0.36 \\
1.78 \\
2.89\end{array}$ & $\begin{array}{r}4 \times 4 \\
4 \times 8 \\
8 \times 8 \\
8 \times 16 \\
16 \times 16\end{array}$ & $\begin{array}{l}0.05 \\
0.10 \\
0.17 \\
0.26 \\
0.50\end{array}$ & $\begin{array}{l}0.06 \\
0.38 \\
0.52 \\
1.29 \\
3.36 \\
\end{array}$ & $\begin{array}{l}0.56 \\
0.57 \\
0.66 \\
0.66 \\
0.80 \\
\end{array}$ & $\begin{array}{r}1.90 \\
2.20 \\
3.65 \\
4.02 \\
12.39 \\
\end{array}$ & $\begin{array}{l}1.00 \\
1.09 \\
1.64 \\
2.06 \\
4.86 \\
\end{array}$ \\
\hline
\end{tabular}

\section{References}

1] S. Anily. and A. Federgruen. "Structured Partitioning Problems," Operations Research. Vol, 13, No. 1, 1991, pp.130-149.

[2] S.H. Bokhari. "Partitioning Problems in Parallel. Pipelined, and Distributed Computing," IEEE Truns. Computers, Vol. 37, No. 1, Jan., 1988, pp. 48-57.

13] T. Bultan. and C. Aykanat, "A New Mapping Heuristic Based on Mean Field Annealing." J. Parallel and Distributed Comp., Vol. 16, 1992, pp. 292-305.

[4] U.V. Catalyurek. arıd C. Aykanat. "Hypergraph-Partitioning Based Decomposition for Parallel Sparse-Matrix Vector Multiplication," submitted for publication in J. Parallel and Distributed Computing.

[5] B. Charny, "Matrix Partitioning on a Virtually Shared Memory Parallel Machine." IEEE Trans. Parallel and Distributed Systems. Vol. 7, No. 4, Apr., 1996, pp. 343-355.

[6] H. Choi, and B. Narahari "Algorithms for Mapping and Partitioning Chain Structured Parallel Computations." Proc. 1991 Int. Conf. on Parallel Processing. pp. I-625-I-628, 1991.

171 G.N. Frederickson. "Optimal Algorithms for Partitioning Trees," Proc. Second ACM-SIAM Symp. Discrete Algorithms. 1991, pp. 168-177.

181 D.M. Gay, "Electronic Mail Distribution of Linear Programming Test Problems," Mathemarical Programming Society COAL Newsletter, 1985.

191 M. Grigni, and F. Manne. "On the Complexity of the Generalized Block Distribution." Proc. 3 rd Int. Workshop on Parallel Algorithms for Irregularly Structured Problems (IRREGULAR'96), 1996, pp. 319-326.

[10] Y. Han. B. Narahari, and H.-A. Choi, "Mapping a Chain Task to Chained Processors," Infor. Proc. Let.,Vol. 44, 1992, pp. 141-148.

[11] P. Hansen, and K.W. Lih. "Improved Algorithms for Partitioning Problems in Parallel. Pipelined and Distributed Computing," IEEE Trans. Computers, vol. 41. No. 6. June. 1992. pp. 769-771.

$12 \mid$ B. Hendrickson, R. Leland, and S. Plimpton, "An Efficient Parallel Algorithm for Matrix-Vector Multiplication," Int. J. High Speed Computing, Vol. 7. No. 1. 1995. pp. $73-88$.

113 M.A. Iqbal. "Approximate Algorithms for Partitioning and Assignment Problems." Tech. Rep. 86-40. ICASE. 1986
[14] M.A. Iqbal, J.H. Saltz, and S.H. Bokhari, "A Comparative Analysis of Static and Dynamic Load Balancing Strategies," Proc. 1986 Int. Conf. on Parallel Processing, 1986, pp. 1040-1047.

[15] M.A. Iqbal, and S.H. Bokhari, "Efficient Algorithms for a Class of Partitioning Problems," Tech. Rep. 90-49, ICASE, 1990.

[16] M.A. Iqbal, "Efficient Algorithms for Partitioning Problems," Proc. 1990 Int. Conf. on Parallel Processing, 1990, pp. III-123-III-127.

[17] M.A. Iqbal, "Approximate Algorithms for Partitioning and Assignment Problems," Int. J. Parallel Programming, Vol. 20, No. 5, 1991, pp. 341-361.

[18] M.A. Iqbal, and S.H. Bokhari, "Efficient Algorithms for a Class of Partitioning Problems," IEEE Trans. Par. and Dist. Systems, Vol. 6, 1995, pp. 170-175.

[19] G. Karypis, and V. Kumar, "A Fast and High Quality Multilevel Scheme for Partitioning Irregular Graphs," Tech. Rep., Dept. of Computer Science, University of Minnesota, 1995.

[20] D.M. Kincaid, D.M. Nicol, D. Shier, and D. Richards, "A Multistage Linear Array Assignment Problem," Oper. Res., Vol. 38. No. 6, 1990, pp. 993-1005.

[21] V. Kumar, A. Grama, A. Gupta, and G. Karypis, Introduction to Paralle Computing. Benjamin/Cummings, 1994.

[22] F. Manne, and T. Sørevik, "Optimal Partitioning of Sequences," J. Algorithms, Vol. 19, 1995, pp. 235-249

[23] F. Manne, and T. Sørevik, "Partitioning an Array onto a Mesh of Processors," Proc. 3rd Int. Workshop on Applied Par. Comp. (PARA'96), 1996, pp. 467-476.

[24] D.M. Nicol, and D.R. O'Hallaron. "Improved Algorithms for Mapping Pipelined and Parallel Computations," IEEE Trans. Computers, Vol. 40, No. 3 , 1991, pp. 295-306.

25] D.M. Nicol, "Rectilinear Partitioning of Irregular Data Parallel Computations," J. Parallel and Disrihuted Computing, Vol. 23, 1994, pp. 119-134.

[26] A.T. Ogielski, and W. Aiello, "Sparse Matrix Computations on Parallel Processor Arrays," SIAM J. Scientific Comp. , Vol. 14, 1993,pp. 519-530.

[27] B. Olstad, and F. Manne, "Efficient Partitioning of Sequences," IEEE Trans. Computers, Vol. 44, No. 11, 1995, pp. 1322-1326.

1281 L.F. Romero, and E.L. Zapata, "Data Distributions for Sparse Matrix Vector Multiplication," Parallel Computing. Vol. 21. 1995, pp. 583 605. 